\title{
New Theory to Understand the Mechanism of Gravitation
}

\author{
Nader Butto \\ Cardiologist, Petah-Tikva, Israel \\ Email: nader.butto@gmail.com
}

How to cite this paper: Butto, N. (2020) New Theory to Understand the Mechanism of Gravitation. Journal of High Energy Physics, Gravitation and Cosmology, 6, 462-472.

https://doi.org/10.4236/jhepgc.2020.63036

Received: June 1, 2020

Accepted: June 27, 2020

Published: June 30, 2020

Copyright $\odot 2020$ by author(s) and Scientific Research Publishing Inc. This work is licensed under the Creative Commons Attribution International License (CC BY 4.0).

http://creativecommons.org/licenses/by/4.0/

(c) (i) Open Access

\begin{abstract}
Gravitation is still the least understood interaction among the fundamental forces of Nature. A new theory that explains the mechanism of gravitation and the origin Newton's laws of gravitation and general relativity and distinguishes between two of the Newton's laws has been proposed. It is shown that the vortex formation created during the Big Bang event is the origin of the gravitational force. The vortex curves the vacuum (space-time) around it, attract and condense energy and dust to its center to form the mass. The gradient pressure in the vortex creates a flow that upon interaction with an object transfers a part of its momentum to the object and pushes it toward the center. The force exercised on the object is equivalent to Newton's second law. The force of attraction between two vortices is equivalent to Newton's third law. The drag force between the energy flow of the vortex and the static vacuum diminishes the gravitational force and is equivalent to the $G$ constant. The proposed theory could provide new interesting insights for a comprehensive understanding of gravitation and represents a theoretical starting point for the engineering of anti-gravitation technology.
\end{abstract}

\section{Keywords}

Vortex Formation, Vacuum Density, Pressure Gradient, General Relativity, Newton Laws of Gravitation, $G$ Constant

\section{Introduction}

Gravity is the most mysterious and still an incompletely understood interaction among the fundamental forces of nature. In fact, the gap in our understanding of gravity is so great that for nearly a century, it has thwarted the ultimate quest of unifying all four fundamental forces into one final explanatory Theory of Everything. 
The universal nature of gravity is demonstrated by the fact that its basic equations closely resemble the laws of thermodynamics and hydrodynamics [1]. So far, there has not been a clear explanation for this resemblance.

Gravity dominates at large distances, but is very weak at small scales. In fact, its basic laws have only been tested up to distances of the order of a millimetre.

Most of the mainstream physics that we are taught are based on Newtonian and Einstein's physics; however, in both these theories, the mechanism giving rise to gravitation is completely unknown. Gravity is also considerably harder to combine with quantum mechanics than all the other forces. The quest for unification of gravity with these other forces of Nature, at a microscopic level, leads to many problems, paradoxes and puzzles. Some problems of the gravitational theory could be, in principle, solved in the framework of extended theories of gravity [2].

Newton's second law of motion defines the relation between the acceleration, force, and mass. In an inertial reference frame, the vector sum of the forces $F$ on an object is equal to the mass $m$ multiplied by the acceleration $a$ of that object: $F$ $=m a$.

Newton's law of motion states that every object in the universe attracts every other object with a force that for any two bodies is proportional to the mass of each object and varies as the inverse square of the distance between them. This statement can be mathematically expressed by the following well-known equation:

$$
F_{g}=G\left(m_{1} \cdot m_{2} / r^{2}\right)
$$

where $m_{1}$ and $m_{2}$ are the interacting masses, $r$ is their relative distance vector, and $G$ is generally assumed to be a universal constant. However, there is no theoretical or mathematical formulation that explains the origin of this equation.

This paper aims to provide a new and unique approach by presenting a satisfying theory to explain the mechanism of gravity. Based on the basic assumption that the universe is immersed in a vacuum with a well-defined density that behaves as a fluid, rotating bodies in liquid generate a drag force and creates vortices that attract other liquid to the center of the vortex. By applying the hydrodynamic laws to calculate the attractive force between two vortices, gives origin to Newton's third law of motion, the interaction between the flow of the vortex and non-rotating body gives origin Newton's second and third laws of motion to the Newton's second law of motion. Therefore, for the physical and mathematical basis for the origin of Newton's second and third laws of motion are described. In addition, the mechanism of vortex formation and the essence of the universal constant $G$ are briefly described, with further details discussed in other papers.

\section{Density of the Vacuum}

By definition, a vacuum has no mass since it has no factor that produces a mass. According to the superfluid theory of the vacuum, the physical vacuum is de- 
scribed as a quantum superfluid which behaves like a fluid with minimal viscosity and with extremely high thermal conductivity. It is a perfect fluid in the sense that it is non-particulate and has no structural memory. If perturbed, it has no tendency to revert to its former physical state.

Furthermore, the first postulate of general relativity states that the source of a gravitational field is the stress-energy tensor of a perfect fluid [3].

This "stress-energy tensor" contains four non-zero components i.e., the density of the perfect fluid and the pressure of the perfect fluid in each of the three physical axes. According to general relativity, a perfect fluid is defined as a fluid with no viscosity or heat conduction.

However, the quantum theory requires the empty space to be filled with particles and anti-particles being continually created and annihilated. This could lead to a net density of the vacuum, which if present, would behave as a cosmological constant. Although there is no consensus about the value of the vacuum density, its value mainly relies on general relativity. The energy density of the vacuum can be measured through astronomical observations that determine the space-time curvature and the expansion of the universe.

It is important to note that the study of the expansion rate of the universe has shown that the universe is close to critical density. Critical density is the value at which the Universe is balanced and expansion is halted.

The density is typically expressed as a fraction of the density required for the critical condition to be fulfilled through the use of a parameter known as omega $(\Omega)$ where $\Omega=\rho / \rho_{\text {critical }}$.

For a value of omega less than 1 (known as an "open universe"), the final fate of the universe is a "cold death". In this case the universe expands forever, albeit at an ever-decreasing rate. For omega greater than 1, the universe is "closed" and will at some point collapse in on itself and end in a "big crunch". For omega equal to 1 , the universe is called "flat"; this universe has a critical density and expansion in halted only after an infinite time. Currently, the estimated sum of the contributions to the total density parameter, $\Omega_{0}$, is $\Omega_{0}=1.02 \pm 0.02$ which indicates that the universe is close to critical density.

The expansion of the universe has been tested using a number of methods, where three of them are mentioned here:

The first one is the Wilkinson Microwave Anisotropy Probe (WMAP) mission completed in 2003, representing a major advance in the precision of determining the expansion of the universe, the Hubble constant, and the calculation of the vacuum density [4].

The second one is using the Baryon Oscillation Spectroscopic Survey (BOSS) [5]. Studying more than 140,000 extremely bright galaxies known as quasars, which serve as a "standard ruler", scientists can map density variations in the universe. By nearly tripling the number of quasars previously studied, as well as implementing a new technique, the scientists were able to calculate the expansion rate to 42 miles (68 kilometers) per second per 1 million light-years with 
greater precision, while looking farther back in time.

The third method is using the infrared camera installed in 2009 on the Hubble Telescope, the astronomical measurements used to calculate the Hubble constant obtained a slightly higher value with narrow error bars. A recent study [6] indicates that $H_{0}=73.24 \mathrm{~km} / \mathrm{s} / \mathrm{Mpc}$ and the cosmological density remains with a small uncertainty calculated as follows:

$$
\rho_{c}=3 H_{0}^{2} / 8 \pi G=11.11( \pm 1.05) \times 10^{-27} \mathrm{~kg} / \mathrm{m}^{3},
$$

where $\rho_{c r}$ represents the critical density, $H$ represents the current value of the Hubble constant, and $G$ represents the gravitational constant.

\section{Hydrodynamics of Vacuum Vortices}

According to the hot Big Bang theory, the observable universe was an "emerging" of space-time from infinitesimal dimensions approximately 13.7 billion years ago. When the Big Bang event occurred, there was nothing except an empty space, a false vacuum, a fluid-like zero-point energy field in a high-energy fluctuation state. Vacuum energy fluctuations are special as the virtual quanta are emitted from and absorbed by the vacuum itself without the presence of real quanta or any other known phenomenon in a state of symmetry. The intrinsic physical feature was "preserved" until the Big Bang that caused a symmetry breaking, primordial-field perturbation, and dynamic flow in the field. Despite a range of different models for the early universe that widely vary in their predictions of the size of these perturbations, all these models predict the creation of black holes with masses ranging from a Planck mass to hundreds of thousands of solar masses [7].

The diversity of temperature and density after the Big Bang resulted in dynamic flow and manifestation of physical effects, such as black holes and stars formation.

Since the Big Bang, the universe has continued to expand, as demonstrated by the gradually increasing distance between our galaxy and external galaxies.

While the universe is expanding, a different region was contracting such that the total energy was preserved; therefore, the expansion of the total universe is accompanied by contraction in some part of it.

This could explain the conflicting observation results of the relative motions of stars and whether they display systematic expansion or contraction [8] [9] [10].

The local area contraction is a type of gravitational force due to vortex formation that condenses the vacuum and causes matter accumulation.

The primordial-field condensation and matter formation is explained by the density wave theory. The density wave theory proposed by Lin and Shu in 1964 explains the spiral-arm structure formation of spiral galaxies. According to this theory, the arms do not comprise matter but are made of regions of greater density with longitudinal compression waves and density fluctuation due to self-gravitation [11].

Vacuum vortices are characterized by power and volume, which may be of 
any magnitude. Each vortex originates from the rotation orbits of another larger vortex.

The energy flow along the galaxy spiral arms creates a turbulent flow and smaller vortices. The field and the gases are drawn down a plug hole that has a point of suction at its center. A cloud of gas, mainly hydrogen, is trapped at the point of suction. It begins to collapse into itself because of its self-gravitational pull and forms the mass of a planetary star.

Gravitation as a push pressure is the origin of Newton's second law.

The pressure gradient in the vortex attracts energy from the vacuum to its core. The flux in such a vortex comprises massless photons. Although photons are zero-rest mass particles, they have the properties of energy and momentum and thus exhibit the property of mass because they travel at the speed of light. Upon colliding with a mass, the photons transfer a fraction of their momentum to that mass and push it toward the center of the vortex with a force proportional to the extension or the volume and density (the mass) of the considered molecule.

In hydrodynamics, the dynamic pressure in the vortex is directly correlated with the medium density. It can be expressed as follows:

$$
P_{d}=\frac{1}{2} \rho v^{2},
$$

where $P_{d}$ is the gradient pressure, $\rho$ is the vacuum density, and $v$ is the velocity of the flow, which in our case is the speed of light.

Consider a sphere immersed in the arm of the vortex with an area that faces the flow. Such a sphere will be pushed to the center of the vortex with a force that is directly proportional to the pressure gradient multiplied by the area of the sphere. Therefore, the force on the sphere can be expressed as follows:

$$
F=P_{d} A,
$$

where $P$ is the gradient pressure and $A$ is the contact area. The flow will face only half of the area of the sphere, so when the area of the sphere is $4 \pi r^{2}$, the pressure will be applied on the area of $2 \pi r^{2}$.

Substituting the dynamic pressure $P_{d}$ in equation 3 by $1 / 2 \rho v^{2}$, the push force on the sphere is obtained as follows:

$$
F=P_{d} A=\frac{1}{2} \rho v^{2} 2 P I r^{2}=\rho v^{2} P I r^{2} .
$$

We then multiply and divide equation 4 by $t$ (time) to obtain

$$
F=\rho \pi r^{2} v t v / t .
$$

Now, the speed $v$ multiplied by time $t$ is equal to space $L$ (length) and the velocity divided by $t$ is equivalent to acceleration a. Therefore, the area $\pi r^{2}$ times space $L$ is equal to volume $Q$.

$$
F=\rho \pi r^{2} L a=\rho Q a .
$$

Note that density multiplied volume is equal to mass, and therefore, we obtain 


$$
F=m a,
$$

which corresponds to Newton's second law of gravitation.

\section{Attraction Force between Two Vortices}

In hydrodynamics, the field reaching the center of the vortex is oriented at an angle of $90^{\circ}$ or perpendicular to the vortex plane. In this vortex, the free surface sharply dips near the axis line, with the depth inversely proportional to $r^{2}$. The shape formed by the free surface is referred to as a hyperboloid or "Gabriel's Horn" and is shown in Figure 1.

In an irrotational vortex flow with a constant fluid density and cylindrical symmetry, the dynamic pressure varies as $P_{\infty}-K / r^{2}$, where $P_{\infty}$ is the limiting pressure that is infinitely far from the axis.

Furthermore, the vacuum with a density of approximately $10 \times 10^{-27} \mathrm{~kg} / \mathrm{m}^{3}$ has viscosity and stiffness and experiences a drag force. Influid dynamics, the drag equation is used to calculate the force of drag experienced by an object due to movement through a fully enclosing medium. In our case, the drag force is related to the dynamic (shear) viscosity of a vacuum that expresses its resistance against shearing flows, where the adjacent layers move parallel to each other at different speeds.

The drag force equation can be expressed as follows:

$$
F_{d}=\frac{1}{2} \rho v^{2} A C_{D},
$$

where $F_{d}$ is the drag force, which by definition is the force component in the direction of the flow velocity, $\rho$ is the mass density of the vacuum, $v=c$ is the relative velocity relative to the vacuum, $A$ is the contact area, and $C_{D}$ is the drag coefficient.

As stated above, $F_{d}$ represents the resistance of the adjacent layers moving parallel to each other at different speeds. The drag force calculated according to the above equation yields a value that is equivalent to that of constant $G$.

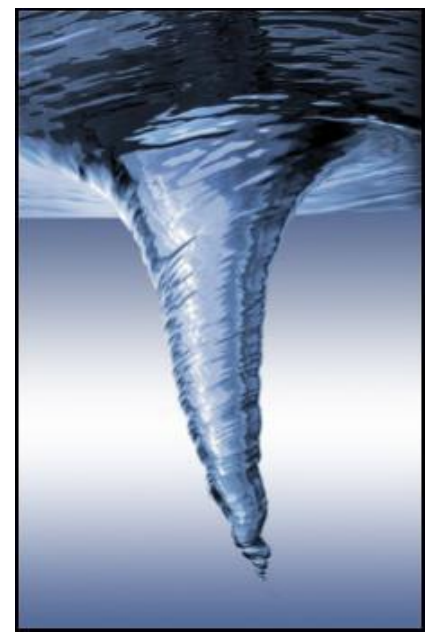

Figure 1. An image illustrating the vortex on a free surface. 
The force of attraction to the center of the vortex is directly related to the pressure gradient, which is equal to the centripetal force:

$$
\frac{1}{2} \rho v^{2}=m v^{2} / r
$$

Then,

$$
\frac{1}{2} \rho=m / r
$$

where $m / r$ is the linear mass density, which is the amount of mass per unit length.

The interaction between two vortices creates tension that attracts one vortex to the other.

The tension on a body can be numerically expressed as follows:

$$
T=m g+m a,
$$

where $T$ indicates tension (Newton), $m$ indicates mass $(\mathrm{kg}), g$ indicates gravitational force $\left(9.8 \mathrm{~m} / \mathrm{s}^{2}\right)$, and a indicates acceleration $\left(\mathrm{m} / \mathrm{s}^{2}\right)$.

In our case, there is no external gravitation force; however, the mass changes according to its distance from the core of the vortex. Therefore, it expressed as linear mass density $(\mathrm{m} / \mathrm{r})$.

The tension in the vortex would be the sum of linear mass density and the force of attraction of the vortex. Therefore, the tension in the vortex can be expressed as follows:

$$
T=m / r+\rho v^{2} P I r^{2}
$$

Since $\rho v^{2} P I r^{2}=F=m a$.

Thus, the tension on the first vortex is:

$$
T=m_{1} / r+m_{1} a
$$

In addition, the tension in the second vortex can be expressed as follows:

$$
m_{2} / r+m_{2} a
$$

Furthermore, the tension between both vortices can be expressed as follows:

$$
T=m_{1} m_{2} / r^{2}+m_{1} a-m_{2} a
$$

In the point of interaction between the two vortices, the linear mass density is cancelled and the acceleration is zero.

Therefore,

$$
T=m_{1} m_{2} / r^{2}
$$

The linear force between the two vortices will be diminished by a force equivalent to the drag force in the vacuum which is, as demonstrated above, equivalent to the value of constant $G$.

Therefore, the attractive force between the two vortices can be expressed as follows:

$$
F=G m_{1} m_{2} / r^{2}
$$


The above equation represents the Newton's law of universal gravitation.

\section{Discussion}

Although even Newton was concerned with the unknown nature of the gravitational force, his basic framework was extremely successful in describing the motion of the planets.

The mechanical theories or explanations of gravitation are attempts to explain the law of gravity with the aid of basic mechanical processes, such as pushes, and without the use of any action at a distance. These theories were developed $16^{\text {th }}-19^{\text {th }}$ century in connection with the aether theories [12].

However, those theories were overthrown because most of them led to an unacceptable amount of drag, which has not yet been observed. Several other models have violated the energy conservation law and are incompatible with modern thermodynamics [13].

In general relativity, the effects of gravitation are ascribed to space-time curvature instead of being attributed to the force and the free-falling objects move along the locally straight paths in curved space-time.

General relativity has experienced considerably successful because of its way of predicting phenomena such as precession of Mercury's perihelion and binary pulsars, warping space-time, gravitational red-shifting of light, the relativistic delay of light, the equivalence principle, the geodetic and frame-dragging effects that have been regularly confirmed. However, general relativity absolutely offers no description of the causation of space-time curvature and there is no mechanism to describe why gravity works the way it does.

Furthermore, it cannot be considered as a complete theory of gravity due to its incompatibility with quantum mechanics.

Besides, there is nothing in Newton Theory or General relativity that explains the origin of energy that produces the gravitational forces. In fact, there is no known energy source to support tremendous energy expenditure that attracts all objects on the surface of our planet for over 4.5 billion years.

Herein, a new gravitation mechanism that explains the essence of gravitation, the origin of Newton laws of gravitation, and the space-time warp of general relativity has been presented.

The proposed theory is based on the fact that the vacuum has a specific density associated with it, making it behave as a superfluid. General relativity also imposes superfluid equations onto gravitational relationships. The imposition of superfluid equations has a considerably significant effect: the speed of the propagation of gravity is thereby made finite, because it propagates at speed of light. The finite transmission speed (and related superfluid properties) represents a significant difference between Newtonian gravity and general relativity.

The mechanism of gravitation relies on the vortices created in the superfluid vacuum. The vortices were created after the Big Bang by the symmetry breaking of the fluctuating vacuum. A superfluid vortex would "warp" space-time, con- 
dense the vacuum density, and create the mass, drag the planet, and maintains its rotation.

According to general relativity, planet rotation is dragged by an unknown force. Such a drag implies that there exists friction in the space-time motion with respect to a mass where inertial dragging occurs. The vortex model explains the mechanism of rotation of the planet as a result of imparting spin on the planet drags the planet mass and causes its rotation. The general relativistic formulations show the requirement of tangential motion when the continuum is assumed to be a superfluid.

The second law of Newton is an expression of the force produced by a vortex pressure gradient that collides with an object and pushes it toward the center of the vortex. The superfluid flow creates a radiation pressure identical to the magnetic radiation pressure that exerts a positive force due to the momentum transferred during the interaction of the waves with the matter (de Broglie standing waves). It acts in a direction same as that of the wave propagation. This is same as the concept of quantum mechanics, by which the attractive force of gravity arises due to exchange of virtual gravitons in the same way as the electromagnetic force arises from exchange of virtual photons [14] [15].

In contrast, Newton's law of motion expresses the tension between two opposite forces created by the vortices. Thus, the gravitational force between two celestial bodies is not caused by their mass but by the tension between the vortices that created the mass and warped space-time.

Finally, since the first formulation of Newton's law of gravitation, there is an open question about the nature of gravitation and the origin of the universal constant of gravitation $G$. This constant is experimentally determined, and it unknown whether there exists an analytical formula for determining the Newtonian constant of gravitation $G$. We found that $G$ is an expression of resistance against the gravitational force in the vacuum due to the drag force of the gravitational flow in the vacuum.

\section{Conclusions}

In this study, we described a new mechanism of gravitation that explains Newton's laws of gravity with Einstein's theory of general relativity. The mass was created after the Big Bang by the gravitational force of the vortices that led to the space-time curvature and condensed the vacuum superfluid to create the mass. Therefore, the mass is rather the effect of the gravitation and not the cause.

The gradient pressure in the vortex creates a flow that applies a force equivalent to the electromagnetic radiation pressure, delivers a fraction of the vortex momentum to that mass, and pushes it toward the center of the vortex. Upon colliding with the mass, this pressure creates the force expressed by Newton's second law.

The calculation of the tension between two vortices leads to the same Newton's law of motion. Therefore, Newton's second law is an expression of push 
radiation force, while Newton's law of motion is the expression of attraction between two vortices.

Finally, the gravitational constant $G$ is an expression of the diminished gravitational value due to the drag force of the superfluid flowing in the vortex (radiation pressure) with an adjacent static vacuum. The calculation of the diminished momentum of the radiation pressure yields a value same as that of the gravitational constant $G$ and was reported in a separate study.

Further research and astronomical observations are needed to confirm the proposed vortex model of gravitation.

\section{Acknowledgements}

The author would like to thank Enago (https://www.enago.com/) for the English language review.

This research did not receive any specific grant from funding agencies in the public, commercial, or not-for-profit sectors.

\section{Conflicts of Interest}

The author declares no conflicts of interest regarding the publication of this paper.

\section{References}

[1] Bardeen, J.M., Carter, B. and Hawking, S.W. (1973) The Four Laws of Black Hole Mechanics. Communications in Mathematical Physics, 31, 161-170. https://doi.org/10.1007/BF01645742

[2] Corda, C. (2009) Interferometric Detection of Gravitational Waves: The Definitive Test for General Relativity. International Journal of Modern Physics D, 18, 2275-2282. https://doi.org/10.1142/S0218271809015904

[3] Oldershaw, R.L. (1987) The Self-Similar Cosmological Paradigm: A New Test and Two New Predictions. Astrophysical Journal, 322, 34-36 https://doi.org/10.1086/165699

[4] Mohr, P.J., Newell, D.B. and Taylor, B.N. (2016) CODATA Recommended Values of the Fundamental Physical Constants: 2014. Journal of Physical and Chemical Reference Data, 45, Article ID: 043102.

[5] Dawson, K.S., et al. (2012) The Baryon Oscillation Spectroscopic Survey of SDSS-III. Astronomical Journal, 145, 4.

[6] https://www.youtube.com/watch?v=E43-CfukEgs

[7] Peebles, P.J.E. and Ratra, B. (2003) The Cosmological Constant and Dark Energy. Reviews of Modern Physics, 75, 559-606.

https://doi.org/10.1103/RevModPhys.75.559

[8] Wilkinson Microwave Anisotropy Probe (2013). http://map.gsfc.nasa.gov/

[9] Riess, A.G., Macri, L.M., et al. (2016) A 2.4\% Determination of the Local Value of the Hubble Constant. The Astrophysical Journal, 826, $31 \mathrm{p}$.

[10] Carr, B.J. (2005) Primordial Black Holes: Do They Exist and Are They Useful?

[11] Taylor, W.B. (1876) Kinetic Theories of Gravitation. Annual Report of the Board of Regents, Smithsonian Institution, 205-282. 
[12] Zenneck, J. (1903) Gravitation. In: Sommerfeld A., Ed., Encyklopädie der Mathematischen Wissenschaften Mit Einschluss Ihrer Anwendungen, Vieweg + Teubner Verlag, Wiesbaden, 25-67. https://doi.org/10.1007/978-3-663-16016-8_2

[13] Randall, L. (2005) Warped Passages: Unraveling the Universe's Hidden Dimensions. ECCO, Bredebro.

[14] Feynman, R.P., Morinigo, F.B., Wagner, W.G. and Hatfield, B. (1995) Feynman Lectures on Gravitation. Addison-Wesley, Boston, MA.

[15] Zee, A. (2003) Quantum Field Theory in a Nutshell. Princeton University Press, Princeton. 